\title{
A Study on Total Serum IgE Levels and Absolute Eosinophil Count in Allergic Rhinitis Patients
}

\author{
Dechu Muddaiahํㅗㄴ, Srinivas Venkatarangaiah² \\ ${ }^{1}$ Department of ENT and Head Neck Surgery, BGS Global Institute of Medical Sciences, Bangalore, Karnataka, India. \\ ${ }^{2}$ Department of ENT and Head Neck Surgery, BGS Global Institute of Medical Sciences, Bangalore, Karnataka, India.
}

\section{ABSTRACT}

\section{BACKGROUND}

Allergic rhinitis is an IgE mediated hypersensitivity disease of the mucous membrane of nasal airway characterized by sneezing, itching, watery nasal discharge and sensation of nasal obstruction.

\section{METHODS}

The aim of the study was to evaluate the total serum IgE levels and absolute eosinophil counts in patients with allergic rhinitis, and rhinitis with asthma. In this study, 160 patients of allergic rhinitis attending ENT OPD were clinically examined and investigated with Total Serum IgE levels and absolute eosinophil counts. Statistical analysis was done using SPSS software.

\section{RESULTS}

160 study subjects with signs and symptoms of allergic rhinitis were investigated with total serum IgE levels and absolute eosinophil count. $46.25 \%$ were males and $53.75 \%$ were females. The patients included in the study were aged 10-60 years. Seasonal symptoms were observed in $44 \%$ of patients while perennial symptoms were experienced by $56 \%$ of the patients. As per ARIA classification, $66.3 \%$ had mild, $6.9 \%$ had moderate and $26.9 \%$ had severe allergic rhinitis. Bronchial asthma was found to be the most common comorbidity present in $13 \%$ of subjects, associated sinonasal polyps which was present in $13.8 \% .71 .9 \%$ had raised Total Serum IgE levels and eosinophilia was present in 59.4\%. A significant relationship exists between total IgE levels and eosinophilia in patients with allergic rhinitis.

\section{CONCLUSIONS}

Allergic rhinitis is an IgE mediated inflammatory disease and raised total serum IgE levels and raised absolute eosinophil count in study subjects with clinical signs and symptoms of rhinitis are likely to correlate with allergic aetiology.

\section{KEY WORDS}

Allergic Rhinitis, Allergic Rhinitis with Asthma, Total Serum IgE levels, Absolute Eosinophil Count
Corresponding Author:

Dechu Muddaiah,

Assistant Professor

Department of ENT, Head Neck Surgery, BGS Global Institute of Medical Sciences, \#67, BGS Health and Education City,

Utarahalli Main Road, Kengeri,

Bangalore, Karnataka, India.

E-mail: dechu23@yahoo.com

DOI: $10.14260 /$ jemds/2020/17

Financial or Other Competing Interests: None.

How to Cite This Article:

Muddaiah D, Venkatarangaiah S. A study on total serum IgE levels and absolute eosinophil count in allergic rhinitis patients. J. Evolution Med. Dent. Sci. 2020;9(02):76-80, DOI:
Submission 14-11-2019,

Peer Review 25-12-2019,

Acceptance 01-01-2020,

Published 13-01-2020. 


\section{BACKGROUND}

Allergic rhinitis is an IgE mediated hypersensitivity disease of the mucous membrane of the nasal airway which is associated with paroxysms of sneezing, itching in the nose, thin watery nasal discharge and sensation of nose block. ${ }^{1}$ Asthma, skin atopy and ethmoidal polyps are the common co morbidities seen in patients with allergic rhinitis. Around 20$30 \%$ of Indian population have nasal allergy and $15 \%$ of those patients develop bronchial asthma. ${ }^{2}$ About 1 in 5 patients attending the outpatient clinic of an ENT practitioner complaint of symptoms related to allergy. Patients of Allergic rhinitis, also have disturbed sleep, feeling of tiredness, mood changes and compromised higher mental functions that will significantly hamper patient's quality of life and work productivity. ${ }^{3}$ These patients may also suffer from allergic conjunctivitis, posterior nasal drip, otitis media with effusion, chronic rhino sinusitis, and in paediatric age group malocclusion of teeth and deformities of face. Allergens found in households like dust mites, pet animals, cockroaches and fungal moulds can trigger an attack of acute allergy. The outdoor allergens are plant pollen, industrial allergens like latex, cigarette smoke, exhaust from vehicles, nitrogen oxide and sulphur dioxide. Clinical examination should follow thorough history. Examination of the nose and paranasal sinus is important to look for anatomical variations, ethmoidal polypi, fungal elements and nasal discharge. ${ }^{4}$

Allergic rhinitis has been classified based on - ARIA (allergic rhinitis and its impact on asthma). Based on the symptom duration, severity and its effect on quality of life patients are categorized as persistent or intermittent and mild, moderate and severe. Patients with intermittent allergic rhinitis have bouts of sneezing, watery rhinorrhoea and itching eyes, and patients with persistent allergic rhinitis have more obstructive symptoms like postnasal discharge, disturbance of smell, nose block, headache and also have co morbidities like bronchial asthma and chronic rhinosinusitis. ${ }^{2}$ Patients diagnosed clinically with allergic rhinitis undergo certain basic investigations like complete blood count with absolute eosinophil count, total Serum IgE levels, nasal smear for eosinophilia, skin prink test, allergen specific estimation of IgE with RAST (Radio Allegro Sorbent test), ELISA (Enzyme Linked Immunosorbent Assay) and radiological investigations like CT scan of the paranasal sinuses. ${ }^{1}$

IgE plays an important role in pathophysiology of allergy. ${ }^{5}$ It is the least abundant immunoglobulin isotope; it accounts for only $0.05 \%$ of the total immunoglobulin concentration. In atopic individuals however IgE levels may be increased by over 1000 times. In atopy the total serum IgE concentration is raised. The IgE levels of a newborn are approximately $0.22 \mathrm{IU} / \mathrm{ml}$. By the time the atopic individual reaches 15 years of age, it will reach the adult value and slowly the total serum IgE levels reduces after the seventh decade due to decrease in the number of identifiable allergens. ${ }^{6}$ The normal serum IgE levels in a non-allergic individual is up to $120 \mathrm{IU} / \mathrm{ml}^{3}$ Several factors influence the
Serum IgE levels like dietary factors, genetics, demography and life style habits. The normal range of eosinophils in peripheral smear is $0-6 \%$, while the range of absolute eosinophil count is 40 to 440 cells/cumm.

The aim of the present study is to determine the total serum IgE levels, and absolute eosinophil counts in allergic rhinitis patients, patients with rhinitis and asthma and to correlate the values with symptomatology for use in the diagnosis and management of allergic rhinitis.

\section{METHODS}

This was a prospective study, where a total number of 160 allergic rhinitis allergic rhinitis patients who reported to the ENT outpatient department from January 2017 till January 2019 were included in the study. The sample size was convenient, and we received 160 patients during the study period. These patients were evaluated in detail on the basis of history, clinical examination and diagnostic nasal endoscopy. Patients with associated bronchial asthma and allergic ophthalmic and dermatological symptoms were included. Patients with symptoms of allergic rhinitis like nasal obstruction, paroxysms of sneezing, watery nasal discharge, posterior nasal discharge and headache were classified into mild, moderate and severe Allergic Rhinitis, based on ARIA classification. All patients were evaluated carefully with clinical examination of ear, nose, throat and Respiratory system. The clinical signs and of allergic rhinitis include bogy oedematous nasal mucosa, turbinate hypertrophy, and thin watery mucous in nose. The features of associated allergic conjunctivitis are lid oedema, congestion of conjunctiva, watering and itching around the eyes. Allergic rhinitis with asthma is characterised by features of allergic rhinitis and wheeze from lungs. Total serum IgE levels were measured using CLIA which was represented as IU/ml. AEC was reported from the total count and differential count and by the direct method using light microscope.

\section{Inclusion Criteria}

Allergic rhinitis based on typical history and clinical features in the age group 10 to 60 -years-old attending outpatient department of BGS Global institute of medical science hospital.

\section{Exclusion Criteria}

- Patients using topical steroidal nasal spray.

- Snuff powder users and pregnant mothers.

- Patients with symptoms of allergic rhinitis who are on antihistamines and since last one month.

\section{Statistical Analysis}

The data was entered in Microsoft Excel 2013 and analysed using SPSS software (V.20.0). Descriptive statistics was used, and results were expressed as percentages, mean and SD. And chi square test is applied for significance difference. Authors assumed significance at $\mathrm{p}$ value $<0.05$. 
RESULTS

In the current study, majority of the patients with allergic rhinitis were in the age group of 21-30 years (40\%), followed by $31-40$ years $(26.9 \%), 11-20$ years $16 \%, 41-50$ years $13.10 \%$ and remaining $3.8 \%$ were in the age group of $51-60$ years. In this study, incidence of allergic rhinitis was 46.25 in males and $53.75 \%$ in females. Seasonal symptoms were observed in $44 \%$ of patients while a persistent symptom throughout the year was experienced by $56 \%$ of patients. Among the study subjects, family history of allergy was present in $40 \%$. Other allergic co morbidities were allergic conjunctivitis in $6 \%$ and atopic dermatitis in $5 \%$. In this study, running nose $(90 \%)$ was the most common presenting complaint in patients with allergic rhinitis, followed by nasal obstruction (85\%), itching and paroxysm of sneezing (76\%). $14 \%$ of subjects who presented with allergic rhinitis had associated wheezing. Epistaxis (4.6\%) and cough (1.7\%) were the least common symptoms in allergic rhinitis.

\begin{tabular}{|c|c|c|}
\hline Age-Group (in Years) & No. of Patients & $\%$ \\
\hline $11-20$ & 26 & 16.20 \\
\hline $21-30$ & 64 & 40.00 \\
\hline $31-40$ & 43 & 26.90 \\
\hline $41-50$ & 21 & 13.10 \\
\hline $51-60$ & 6 & 3.80 \\
\hline Total & 160 & 100.0 \\
\hline \multicolumn{3}{|c|}{ Table 1. Distribution of the Study Population Based on Age } \\
\hline
\end{tabular}

\begin{tabular}{|c|c|c|}
\hline Gender & No. of Patients & $\mathbf{\%}$ \\
\hline Female & 86 & 53.75 \\
\hline Male & 74 & 46.25 \\
\hline Total & 160 & 100.0 \\
\hline \multicolumn{2}{|c|}{ Table 2. Gender-Distribution of Study Population } \\
\hline
\end{tabular}

\begin{tabular}{|c|c|c|}
\hline Bronchial Asthma & No. of Patients & $\mathbf{\%}$ \\
\hline Yes & 21 & 13.1 \\
\hline No & 139 & 86.9 \\
\hline Total & $\mathbf{1 6 0}$ & $\mathbf{1 0 0 . 0}$ \\
\hline \multicolumn{2}{|c|}{ Table 3. Patients of Allergic Rhinitis with Bronchial Asthma } \\
\hline
\end{tabular}

\begin{tabular}{|c|c|c|}
\hline Allergic Rhinitis & No. of Patients & $\mathbf{\%}$ \\
\hline Mild & 106 & 66.3 \\
\hline Moderate & 12 & 7.2 \\
\hline Severe & 42 & 26.5 \\
\hline Total & $\mathbf{1 6 0}$ & $\mathbf{1 0 0 . 0}$ \\
\hline Table 4. Classification of Allergic Rhinitis Based on Severity \\
\hline
\end{tabular}

\begin{tabular}{|c|c|c|}
\hline IgE & No. of Patients & $\mathbf{\%}$ \\
\hline$<=100$ & 45 & 28.1 \\
\hline$>100$ & 115 & 71.9 \\
\hline \multicolumn{2}{|c|}{ Table 5. Raised Serum IgE Levels in Study Subjects } \\
\hline
\end{tabular}

\begin{tabular}{|c|c|c|}
\hline AEC & No. of Patients & $\%$ \\
\hline$<440$ cells/cumm & 65 & 40.6 \\
\hline$>440$ cells/cumm & 95 & 59.4 \\
\hline \multicolumn{2}{|c|}{ Table 6. Raised Absolute Eosinophil Count in Study Subjects } \\
\hline
\end{tabular}

Based on severity of symptoms majority that is $66.3 \%$ had mild, $6.9 \%$ had moderate and $26.9 \%$ had sever grade of allergic rhinitis. $13.8 \%$ of study subjects had associated sinonasal polyps and $13 \%$ had rhinitis with bronchial asthma. The total serum IgE levels were raised in $71.9 \%$ of study subjects, and the absolute eosinophil count was raised in
59.4\%. The association of raised serum Ig E levels with allergic rhinitis is significant with $p$ value $=00010766$, and the association between allergic rhinitis and absolute eosinophil count has $\mathrm{p}$ value $=0.176665$.

\section{DISCUSSION}

The aim of the study was to estimate the total serum IgE levels and look for eosinophilia in allergic rhinitis patients and to determine the association between these parameters and allergy. We considered serum IgE levels of $100 \mathrm{IU} / \mathrm{ml}$ as the normal cut of value. The significant finding of this study was a significant association between raised total serum IgE levels and allergic rhinitis, angelo et al in their study on 217 patients found the presence of elevated serum IgE levels was associated with severity of allergic rhinitis. ${ }^{7}$ As per a study done by Halonen et al a strong relationship is present between atopic individuals and serum IgE levels and eosinophilia, and IgE levels provide a better clue to allergic etiology than skin prick test ${ }^{2}$. According to Yamada et al detecting raised serum specific IgE can be considered a prerequisite for diagnosis of allergic diseases and other atopic diseases. ${ }^{8}$

In our study of 160 patients of diagnosed allergic rhinitis, the IgE levels were elevated in $71.9 \%$ of patients, it was observed that $33 \%$ had raised levels more than 1000 IU which was seen in patients with associated co morbidities like sinonasal polyps and rhinitis with asthma. There was a significant association between allergic rhinitis and raised serum total IgE levels. As per the study by V S Chowdary et $\mathrm{al}^{6}$ the total IgE levels in their study ranged from $10 \mathrm{IU} / \mathrm{ML}$ to $380 \mathrm{IU} / \mathrm{ml}$ with a mean of $180 \mathrm{IU} / \mathrm{ml}$ in patients with respiratory allergy.

In our study $13.8 \%$ of study subjects had associated sinonasal polyps and $13 \%$ had rhinitis with bronchial asthma as compared to a study done by Raheleh Shokouhi Shoormasti et al where the frequency of allergic rhinitis with asthma was $12.5 \%$, and allergic conjunctivitis was $43.6 \%{ }^{9}$ The general presumption that allergic rhinitis is associated with peripheral eosinophilia did not correlate with our finding. 59.4\% patients with diagnosed allergic rhinitis had raised absolute eosinophil count suggesting eosinophilia but the association was not statistically significant. Globally the prevalence of Allergic rhinitis in the general population is about $10-40 \%$. Severity of allergic rhinitis was found to have a significant effect on sleep, performance in workplace and quality of life. ${ }^{10}$ Unlike what was considered previously, allergic rhinitis not only affects the nose and paranasal sinuses, but presently there is evidence that it may be a part of a systemic airway pathology which involves both the upper and lower respiratory tract. Several anatomical, physiological and immunological similarities are present between the upper and lower respiratory tract. Allergic rhinitis usually coexists with bronchial asthma, as there is combined inflammatory disease of both upper and lower airway. Whenever the upper respiratory tract is exposed to the allergen, IgE mediated inflammatory response occurs not 
only inth nasal passage but also in the lower respiratory tract.[11,12]

Patients with perennial, persistent allergic rhinitis, who are allergic to allergens like dust mite are more susceptible to develop Bronchial Asthma, than patients with seasonal allergic rhinitis who are allergic to pollens. Genetically predisposed individuals are more prone to develop allergic rhinitis and asthma. The susceptibility to develop allergic rhinitis or asthma is seen in genetically predisposed individuals. Allergens from house dust mite, furred pets (Cats and Dogs), mice, cockroaches, and fungi comprise the most common indoor allergens implicated in patients with atopic disease. ${ }^{13}$

Allergic rhinosinusitis is classified as seasonal allergic rhinitis or perineal allergic rhinitis based on allergic sensitization to seasonal or perineal allergens. Seasonal allergic rhinitis patients are allergic to seasonal pollens while as the common allergens causing perennial allergic rhinitis are dust mites, dander of pet animals, insects, cockroaches and fungal moulds. The pathophysiology of Allergic rhinitis is characterized by cellular response of inflammation, where there is release of chemokines and cytokines, trans endothelial migration of polymorphonuclear cells, activation of eosinophils, mast cells and $\mathrm{T}$ cells, release of chemical mediators of inflammation like histamine and leukotrienes. Whenever the nasal mucosa is exposed to an inciting allergen like faecal matter of dust mites, cockroach residues, dander of animals, pollens and plant moulds, the IgE mediated inflammatory response results in infiltration of nasal mucosa by several inflammatory cells, mast cells, CD4 T cells, B cells, macrophages and eosinophils. The $\mathrm{T}$ helper cells that infiltrate the nasal mucosa release cytokines (e.g., interleukin [IL]-3, IL-4, IL-5, and IL-13) that activates the plasma cells to produce immunoglobulin E (IgE). The allergen specific IgE cross link with allergen bound mast cells which results in a trigger for release of chemical mediators like histamine and leukotrienes, that bring about dilatation of arterioles, increase in vascular permeability and causes symptoms like itching, watery nasal discharge, increased secretion of mucus and contraction of smooth muscles in the lungs. ${ }^{[12,14]}$ In the early phase of immune response the chemical mediators and cytokines are released. The late phase of inflammatory response occurs over the next 4-8 hours which is a predominantly cellular inflammatory response which results in nasal congestion and blockade which often persists. ${ }^{11}$

Even though allergic rhinitis is classified as perennial allergic rhinitis and seasonal allergic rhinitis, not all the patients will completely fit into the classification. For example, certain allergens, like pollen, may be seasonal in cold climate, but perennial and present all through the year in hot climate, and patients with multiple "seasonal" allergies may suffer from symptoms throughout the year. ${ }^{14}$ Presently as per ARIA, allergic rhinitis is now classified according to duration of symptoms as (intermittent or persistent) and based on severity of symptoms and its effect on sleep and life style as (mild, moderate or severe). ${ }^{15}$ As per the (ARIA) Allergic Rhinitis and its Impact on Asthma guidelines patients with symptoms less than 4 days per week or for less than 4 consecutive weeks are classified as "intermittent" allergic rhinitis and patients with symptoms that are present more than 4 days/week and for more than 4 consecutive weeks as "persistent" allergic rhinitis. ${ }^{15}$

If patients have good sleep and are able to perform daily activities at workplace and school, they are classified as mild and patients are classified as moderate/severe if their symptoms are significantly affect sleep or daily living activities, and/or if symptoms are considered bothersome. Classifying patients based on ARIA is important for appropriate management of individual patients. Allergic rhinitis is usually diagnosed on the basis of allergic rhinitis is based on typical history of allergic symptoms. Runners and Sneezers have paroxysmal bouts of sneezing, mucoidal nasal discharge and postnasal drip, itching of nose and diurnal variation of symptoms. Blockers do not sneezing, but have thick mucoidal nasal discharge with postnasal drip and severe nasal blockage which is present throughout the day.

\section{Diagnosis and Investigations}

Not only proper history and ENT examination are required for establishing the diagnosis of allergic rhinitis, but certain diagnostic tests are necessary to confirm that underlying rhinitis is due to allergy. There are In vivo and in vitro tests which are used to diagnose allergic diseases, absolute eosinophil count estimation and measurement of total IgE and allergen specific IgE in serum and skin prick test is done. A radiological investigation like CT scan of Paranasal sinuses is required to look for infective and neoplastic pathologies. All patients with associated bronchial asthma should undergo a pulmonary function test. Subcuticular Skin-prick test(Immediate hypersensitivity testing), is a in vivo test to determine immediate ( $\mathrm{IgE}$ mediated) hypersensitivity to specific allergens. This test is highly sensitive and is used as the primary method for identifying specific allergens. In this test a drop of a commercial extract solution of a specific allergen is placed on the unprepared skin of the medial aspect of forearm, then a lancet is used to prick the skin through the drop to introduce the extract into the epidermis, care is taken not to penetrate the dermis. If the test is positive within 1520 min, a wheal-and-flare response will occur. Allergens relevant to the patient's environment (e.g., pollen, animal dander, moulds and house dust mites) are tested. Other diagnostic tests are assessment of allergen specific serum IgE and total serum IgE levels. RAST Radioallergosorbent tests are used to assess the amount of specific IgE to individual allergens in a sample of blood. ${ }^{4}$ The amount of IgE produced to a particular allergen correlates approximately with the sensitivity to the allergen. The other diagnostic tests are absolute eosinophil count and taking nasal smear for eosinophilia.

Providing symptomatic relief is the aim in treating allergic rhinitis. Along with pharmacotherapy for allergic rhinitis, lifestyle modifications like avoidance of allergen exposure, normal saline nasal douching is also necessary. Patients are treated with oral antihistaminic, topical steroidal sprays, combination intranasal corticosteroids/ antihistamine sprays, oral and topical decongestants, leukotriene receptor antagonists and immunotherapy. Certain patients with associated sinonasal polyps may need oral corticosteroids as well. In immunotherapy allergens are administered subcutaneously in gradual increasing 
concentration, until a dosage where patient develops immunological tolerance to the allergen. Patient with associated bronchial asthma are treated for the same. Environmental and social factors should be optimized to allow the patient to lead a normal life. To treat coexisting upper and lower airway disease a combined strategy is ideally used.

\section{CONCLUSIONS}

Based on our study, it is recommended that total serum IgE levels and absolute eosinophil counts be done in all cases of rhinitis as it is cost effective and suggests atopy.

\section{REFERENCES}

[1] Rudrappa S, Kumar R, Kumar V. Study of eosinophil count in nasal smear and peripheral blood smear in children with allergic rhinitis. International Journal of Contemporary Paediatrics 2019;6(3):1158-62.

[2] Varshney J, Varshney H. Allergic rhinitis: an overview. Indian Journal of Otolaryngology and Head Neck Surgery 2015;67(2):143-9.

[3] Meltzer EO. Quality of life in adults and children with allergic rhinitis. Journal of Allergy Clinical Immunology 2001;108(1 Suppl):S45-S53.

[4] Quillen DM, Feller DB. Diagnosing rhinitis: allergic vs. non-allergic. American Famly Physician 2006;73(9):1583-90.

[5] Choi BG, Lee YW, Chole YB, et al. Total serum immunoglobulin E level and specific allergens in adults with skin diseases. Indian Journal of Dermatology Venerology Leprol 2018;84(2):148-52.

[6] Chowdary VS, Vinaykumar EC, Rao JJ, et al. Study on serum IgE and eosinophils in respiratory allergy patients. Indian J Allergy Asthma Immunol 2003;17(1):21-4.

[7] Burbank AJ, Sood AK, Kesic MJ, et al. Environmental determinants of allergy and asthma in early life. J Allergy Clinical Immunology 2017;140(1):1-12.

[8] Dykewicz MS, Hamilos DL. Rhinitis and sinusitis. J Allergy Clin Immunol 2010;125(2 Suppl 2):S103-15.

[9] Small P, Frenkiel S, Becker A, et al. Rhinitis: a practical and comprehensive approach to assessment and therapy. J Otolaryngol 2007;36(Suppl 1):S5-27.

[10] Bourdin A, Gras D, Vachier I, et al. Upper airway 1: allergic rhinitis and asthma: united disease through epithelial cells. Thorax 2009;64(11):999-1004.

[11] Lee P, Mace S. An approach to allergic rhinitis. Allergy Rounds 2009;1:1.

[12] Bousquet J, Khaltaev N, Cruz AA, et al. Allergic rhinitis and its impact on asthma (ARIA) 2008 update (in collaboration with the World Health Organization, GA (2) LEN and AllerGen). Allergy 2008;63(Suppl 86):8-160.

[13] Corsico AG, De Amici M, Ronzoni V, et al. Allergen specific immunoglobulin $\mathrm{E}$ and allergic rhinitis severity. Allergy and Rhinology 2017;8(1):e1-e4.

[14] Yamada K, Ohashi Y, Tanaka A, et al. Clinical evaluation of lumiward immunoassay system for detection of specific IgE associated with allergic rhinitis. Acta Otolaryngologica - Supplements 1998;538:169-77.

[15] Shoormasti RS, Pourpak Z, Fazlollahi MR, et al. The prevalence of allergic rhinitis, allergic conjunctivitis, atopic dermatitis and asthma among adults of Tehran. Iran Journal of Public Health 2018;47(11);1749-55. 